\title{
Comprehensive Evaluation and Treatment Modalities of Crooked Nose
}

\author{
SHOEIB M.A., M.D. \\ The Department of Plastic Surgery, Faculty of Medicine, Sohag University, Sohag, Egypt
}

\begin{abstract}
Background: The deviated nose is one that varies from the straight vertical orientation of the face. Correction of this deformity presents a challenge because it represents both a functional problem (airway obstruction) and an aesthetic problem must be addressed.

Patient and Methods: From December 2007 to December 2017, 47 patients with crooked nose were operated; 26 males and 21 females, aged from 18 years to 35 years, with the mean age were 26.5 years.

Results: Different techniques were applied depending on deformity type and severity including; solitary osteotomy and septorhinoplasty. Four patients needed iliac bone grafts for deformity correction.

Conclusion: Absolute correction of the crooked nose is a difficult task because of the complexity of the structural defect, the highly visible, well-defined subunits of the nasal dorsum, the need for highly skilled surgeon and the tendency for recurrence.
\end{abstract}

Key Words: Crooked nose - Comprehensive - Treatment modalities.

\section{INTRODUCTION}

The deviated nose is one that varies from the straight vertical orientation of the face. Correction of this deformity presents a challenge because it represents both a functional problem (airway obstruction) and an aesthetic problem must be addressed [1-4]. The anatomic basis of the deviation may be related to either bony pyramid abnormality, septal deformity, or a combination of both [5-7]. The cause may be congenital or acquired secondary to previous trauma or surgery. Severe nasal injuries involving both septal and asymmetric bony abnormalities present a particularly difficult challenge for the surgeon [8]. Severely deviated noses almost always include major septal deformity as a component [9].

To attain good aesthetic and functional results consistently in correction of the deviated nose thorough understanding of nasal anatomy [10] and physiology with accurate preoperative assessment and intra-operative diagnosis and understanding of cartilage physiology and healing are required [11-13]. A major septal deformity almost always accompanies the crooked nose deformity and impairs both breathing function and the aesthetic appearance [14].

To achieve optimum correction of the crooked nose deformity, the septum must be the target of the treatment. The goal of this article is to present our current principles for correcting the deviated nose both aesthetically and functionally. The aesthetic goals included straightening the dorsum, defining the tip to enhance facial balance, and correcting bony asymmetries. Functionally, the goals were directed at improving the nasal airway, including straightening the septum, and restoring the nasal valve integrity. In selected cases an iliac bone graft was used to replace the deviated septum.

\section{PATIENTS AND METHODS}

From December 2007 to December 2017, 47 patients with crooked nose were operated; 26 males and 21 females, aged from 18 years to 35 years, with the mean age were 26.5 years.

Detailed history was taken with careful physical examination, photographic documentations, preoperative planning and patient counseling; regarding operative technique and possible complications were performed in all cases. A written informed consent was assigned by all patients participating in the study before surgery.

All cases were operated under general hypotensive anesthesia.

Air way obstruction, if present, was addressed and taken in consideration before surgery. Nasal straightening was achieved by either osteotomy alone in cases of solitary bony $\mathrm{C}$ shaped deformity, or by septorhinoplasty in cases of $\mathrm{S}$ shaped bony and cartilaginous deformity. 
In certain cases total replacement of the septum by using an iliac bone graft was done to achieve full correction of the septal deformity (Fig. 3).

\section{Surgical technique:}

1- Solitary osteotomy (Fig. 1).

2- Septorhinoplasty (Fig. 2).

An open approach with a transcolumellar inverted V-shape incision was connected to bilateral marginal incisions. The osseocartilaginous framework was exposed, and the septal mucoperichondrial flaps were elevated, beginning from the anterior septal angle. The septum was freed from the extrinsic forces of the deformed nasal bones and from the upper and lower lateral cartilages. The cartilaginous and bony septum was harvested, leaving at least $10 \mathrm{~mm}$ of the L-strut. The dorsal septal cartilage was first scored vertically. Clocking sutures of 4-0 Polydioxanone were placed caudocephalically on the convex side and cephalocaudally on the concave side between the septum and upper lateral cartilages. When the sutures were tightened, they unfurled the dorsal septum and secured it in the midline. Cartilage memory was directed with the extramucosal stents and spreader grafts anteriorly. Apart from correcting dorsal deformities, any caudal L-strut deformities were corrected by dividing the connection with the anterior nasal spine, removing excess cartilage, and fixing them securely in position by using two to three 4/0 Prolene stitches.

All patients were followed-up for at least 6 months post-operatively with photographic documentation and reporting detected complications.
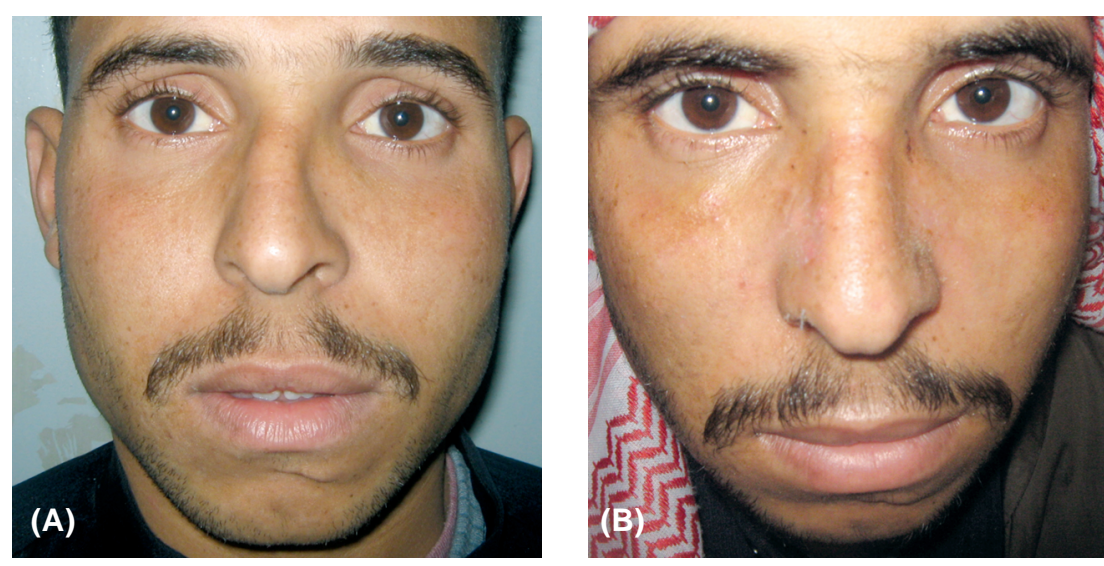

Fig. (1): Male, 25ys (A) Pre-operative, (B) Post-operative after solitary osteotomy.
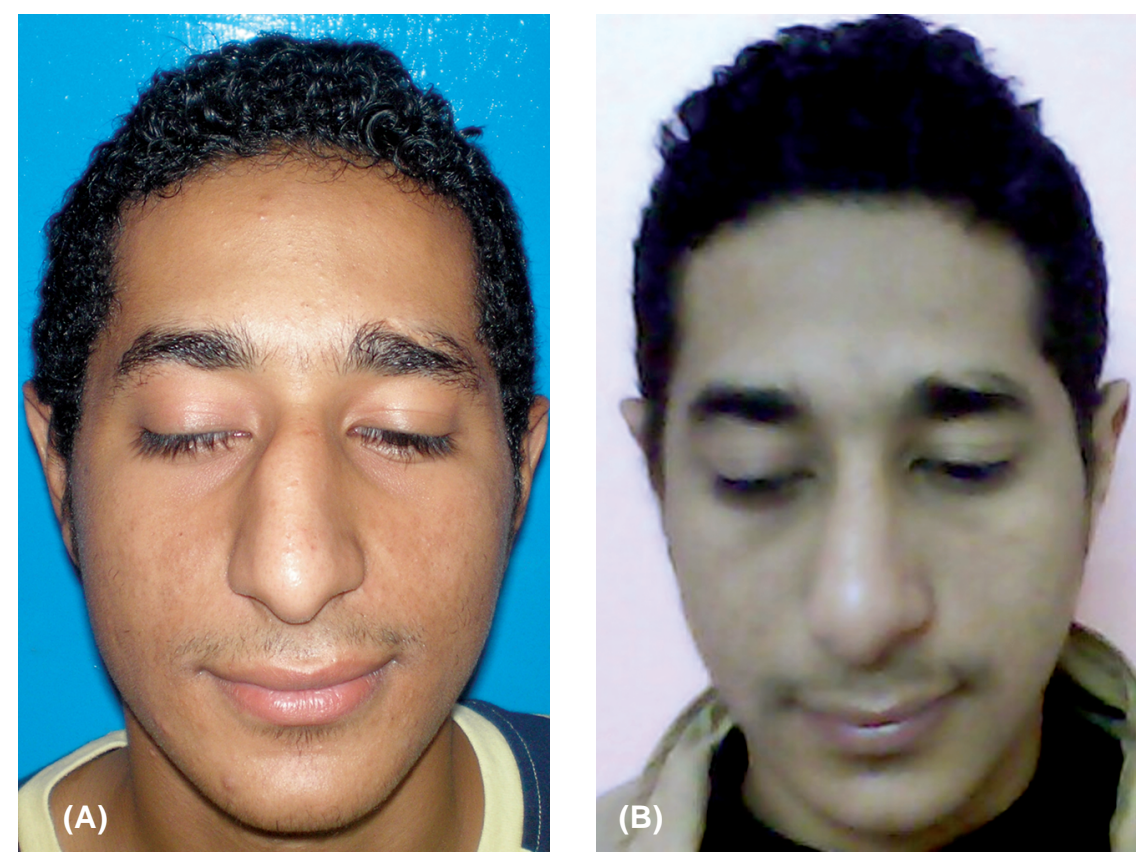

Fig. (2): Male, 22ys (A) Pre-operative, (B) Post-operative after septorhinoplasty. 

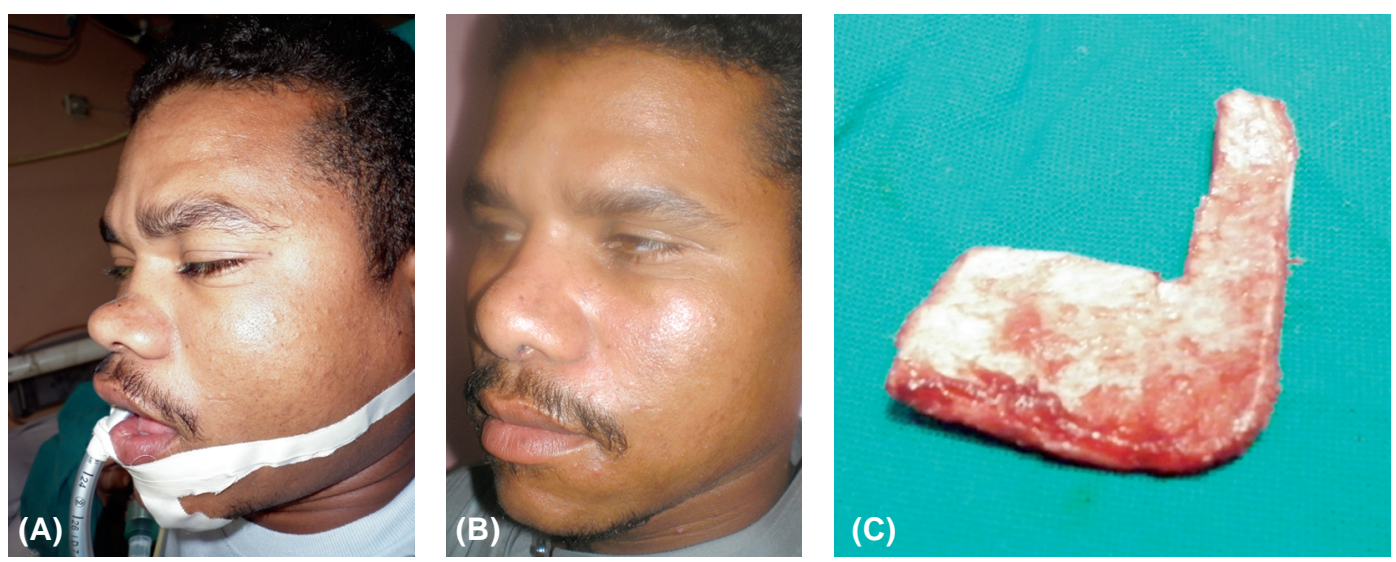

Fig. (3): Male, 28ys (A) Pre-operative, (B) Post-operative after septorhinoplasty and total replacement of the septum by iliac bone graft, $(\mathrm{C})$ Bone graft.

\section{RESULTS}

This study included 47 patients, 26 males and 21 females with their ages ranged between 18 to 35 years. The mean age of presentation was 26.5 years.

Thirty eight patients gave history of nasal trauma as an underlying cause of the deformity, of which 29 cases were related to road traffic accidents assaults and 9 cases had a history of assault. The remaining 9 patients declared that the deformity was present since birth. Patients with acquired crooked nose deformity were presented at variable durations.

Since nasal trauma, ranged from 3 months up to 17 years. The type and severity of the crooked nose deformity were variable among the cases; 21 patients had C-shaped deformity (44.7\%), 18 patients had S-shaped deformity $(38.3 \%)$ and 8 patients were presented with straight line deformity $(17 \%)$.

Pre-operative assessment revealed that the deformity was bony in 16 patients (34\%), cartilaginous in 12 patients $(25.6 \%)$ and combined in 19 patients $(40.4 \%)$.

Different techniques were applied depending on deformity type and severity including; solitary osteotomy and septorhinoplasty. Four patients needed iliac bone grafts for deformity correction.

Seventeen patients developed complications (36\%) in the form of residual deviation; 4 cases (23.5\%), defective breathing; 5 cases $(29.4 \%)$, graft resorption; 2 cases (11.7\%). Minor complications including wound infection, oedema and mild hematoma were detected in remaining cases.
Patient satisfaction was generally adequate; 40 out of 47 patients $(85.1 \%)$.

\section{DISCUSSION}

Crooked nose may be congenital or acquired secondary to previous trauma or surgery [15]. In our study, the majority of cases $(80.85 \%)$ were acquired mostly due to road traffic accidents because of high frequency of road traffic accidents in our community.

Delayed age of patients' presentation in the study was attributed to lack of education and poor socioeconomic status.

Deformities of the bony pyramid, the nasal septum or both are the underlying cause of crooked nose deformity [5-7]. This study revealed that the combined deformity of both the bony pyramid and nasal septum was the underlying cause in a great proportion of study cases.

Absolute correction of crooked nose deformity is difficult to be achieved and requires experience with the conventional procedures including septoplasty, osteotomies and grafting. Numerous techniques were described in review of literature [15]. In this study 2 main techniques have been highlighted; solitary osteotomy and open septorhinoplasty with different procedures were carried out aiming at achieving optimum correction.

Unilateral osteotomy is safe and effective procedure for correction of selected cases of crooked nose deformity with external bony deviation with the advantages of minimal surgical trauma, minimal intraoperative bleeding, minimal post-operative edema and low risk of recurrence. It also avoids 
intrinsic destabilization of the bony framework. Bilateral osteotomy is associated with more complications including excessive bleeding, prolonged hematoma, bilateral ecchymosis and nasal obstruction $[\mathbf{1 5 , 1 6 ]}$. In this study, solitary osteotomy (either unilateral or bilateral) was applied mainly for patients with C-shaped crooked nasal deformity with good outcome.

Severe cases require extracorporeal septal replacement which involves resection of the affected part of the septum with either remodeling and returning it or replacing it with an alternative material [17]. Four patients needed total replacement of the septum using an iliac bone graft for full correction of the septal deformity.

Extracorporeal septoplasty is the procedure of choice for correcting complex nasal septal deformities however, it may destabilize the keystone area and cause dorsal notching or saddling. Moreover, it is technically demanding procedure $[\mathbf{1 8 , 1 9 ]}$. Cartilage memory is an essential factor contributing to deformity recurrence. It can be overcome by cartilage straightening through L-strut cutting and suture technique [20]. In our study, dorsal L-strut cutting and suturing with using spreader graft was applied to patients with severe septal deformities with good outcome.

Defective breathing detected post-operatively in study patients can be attributed to excessive narrowing induced by bilateral osteotomies.

\section{Conclusion:}

Crooked nose deformity is a challenging problem which may be congenital or acquired. Accurate pre-operative assessment, knowledge of the structural anatomy of the nose and proper selection of the most suitable operative technique with awareness of its long-term consequences are the cornerstones for optimum management.

Treatment plan should be individualized based on deformity type, severity and underlying cause (deviated bony pyramid, deviated septum or combination of both).

Absolute correction of the crooked nose is a difficult task because of the complexity of the structural defect, the highly visible, well-defined subunits of the nasal dorsum, the need for highly skilled surgeon and the tendency for recurrence.

\section{REFERENCES}

1- Brain D.J.: The management of the deviated nose. J. Laryngol. Otol., 95: 471, 1981.

2- Dingman R.O. and Natvig P.: The deviated nose. Clin. Plast. Surg., 4: 145, 1977.

3- Johnson C.M., Jr. and Anderson J.R.: The deviated nose: Its correction. Laryngoscope, 87: 1680, 1977.

4- Planas J.: The twisted nose. Clin. Plast. Surg., 4: 55, 1977.

5- Bernstein L.: Submucous operations on the nasal septum. Otolaryngol. Clin. North Am., 6: 675, 1973.

6- Edwards N.: Septoplasty: Rational surgery of the nasal septum. J. Laryngol. Otol., 89: 875, 1975.

7- McKinney P. and Shively R.: Straightening the twisted nose. Plast. Reconstr. Surg., 64: 176, 1979.

8- Dingman R.O.: Correction of nasal deformities due to defects of the septum. Plast. Reconstr. Surg., 18: 291, 1956.

9- Rohrich R.J. and Adams W.P. Jr.: Late salvage of nasal injuries. Oper. Tech. Plast. Surg., 5: 342, 1998.

10- Converse J.M.: Corrective surgery of nasal deviations. Arch. Otolaryngol., 52: 671, 1950.

11- Fry H.: Interlocked stresses in human nasal septal cartilage. Br. J. Plast. Surg., 19: 276, 1966.

12- Fry H.: Nasal skeletal trauma and the interlocked stresses of the nasal septal cartilage. Br. J. Plast. Surg., 20: 146, 1967.

13- Gibson T. and Davis W.B.: The distortion of autogenous cartilage grafts: Its cause and prevention. Br. J. Plast. Surg., 10: 257, 1958.

14- Rohrich R.J., Gunter J.P., Deuber M.A., et al.: The deviated nose: Optimizing results using a simplified classification and algorithmic approach. Plast. Reconstr. Surg., 110: 1509-1523, 2002.

15- Seneldir S., Korkut A.Y., Coskun B.U., et al.: Unilateral Osteotomies for External Bony Deviation. J. Craniofac. Surg., 21: 1890-1893, 2010.

16- Fanous N.: Unilateral osteotomies for external bony deviation of the nose. Plast. Reconstr. Surg., 100: 115123, 1997.

17- Murrell G.L., Requena R. and Wall M.P.: Septal replacement for severe anterior septal deviation. Am. J. Otolaryngol., 21: 147-152, 2000.

18- Gubisch W.: Extracorporeal septoplasty for the markedly deviated septum. Arch. Facial Plast. Surg., 7: 218-226, 2005.

19- Andre R.F. and Vuyk H.D.: Reconstruction of dorsal and/or caudal nasal septum deformities with septal battens or by septal replacement: An overview and comparison of techniques. Laryngoscope, 116: 1668-1673, 2006.

20- Song H.M., Kim J., Lee B., et al.: Deviated Nose Cartilaginous Dorsum Correction Using a Dorsal L-Strut Cutting and Suture Technique. Laryngoscope, 118: 981986, 2008. 